\title{
TAK1-regulated expression of BIRC3 predicts resistance to preoperative chemoradiotherapy in oesophageal adenocarcinoma patients
}

G Piro $^{1,2,3}$, S Giacopuzzi ${ }^{3,4}$, M Bencivenga ${ }^{3,4}$, C Carbone ${ }^{1,3}$, G Verlato ${ }^{5}$, M Frizziero ${ }^{3,6}$, M Zanotto $^{1,3}$,

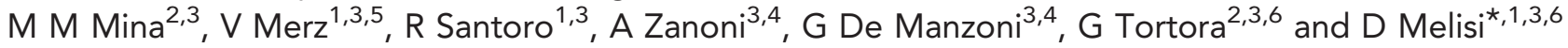

${ }^{1}$ Digestive Molecular Clinical Oncology Research Unit, Università degli studi di Verona, Verona, Italy; ${ }^{2}$ Laboratory of Oncology and Molecular Therapy, Department of Medicine, Università degli studi di Verona, Verona, Italy; ${ }^{3}$ Comprehensive Cancer Center, Azienda Ospedaliera Universitaria Integrata, Verona, Italy; ${ }^{4}$ General and Upper G.I. Surgery Division, Department of Surgery, Azienda Ospedaliera Universitaria Integrata, Verona, Italy; ${ }^{5}$ Section of Epidemiology and Medical Statistics, Department of Public Health and Community Medicine, Università degli studi di Verona, Verona, Italy and ${ }^{6}$ Medical Oncology Unit, Azienda Ospedaliera Universitaria Integrata, Verona, Italy

Background: About $20 \%$ of resectable oesophageal carcinoma is resistant to preoperative chemoradiotherapy. Here we hypothesised that the expression of the antiapoptotic gene Baculoviral inhibitor of apoptosis repeat containing (BIRC) 3 induced by the transforming growth factor $\beta$ activated kinase 1 (TAK1) might be responsible for the resistance to the proapoptotic effect of chemoradiotherapy in oesophageal carcinoma.

Methods: TAK1 kinase activity was inhibited in FLO-1 and KYAE-1 oesophageal adenocarcinoma cells using (5Z)-7-oxozeaenol. The BIRC3 mRNA expression was measured by qRT-PCR in 65 pretreatment frozen biopsies from patients receiving preoperatively docetaxel, cisplatin, 5-fluorouracil, and concurrent radiotherapy. Receiver operator characteristic (ROC) analyses were performed to determine the performance of BIRC3 expression levels in distinguishing patients with sensitive or resistant carcinoma.

Results: In vitro, (5Z)-7-oxozeaenol significantly reduced BIRC3 expression in FLO-1 and KYAE-1 cells. Exposure to chemotherapeutic agents or radiotherapy plus (5Z)-7-oxozeaenol resulted in a strong synergistic antiapoptotic effect. In patients, median expression of BIRC3 was significantly $(P<0.0001)$ higher in adenocarcinoma than in the more sensitive squamous cell carcinoma subtype. The BIRC3 expression significantly discriminated patients with sensitive or resistant adenocarcinoma (AUC$\mathrm{ROC}=0.7773$ and 0.8074 by size-based pathological response or Mandard's tumour regression grade classifications, respectively).

Conclusions: The BIRC3 expression might be a valid biomarker for predicting patients with oesophageal adenocarcinoma that could most likely benefit from preoperative chemoradiotherapy.

Cancers of the oesophagus and of the oesophagogastric junction represent a major health problem worldwide, ranking seventh among leading causes of cancer-related death (Siegel et al, 2014). A multimodal approach consisting of preoperative combined chemoradiotherapy followed by surgery has been accepted as one of the standard of care for the treatment of the T2 or greater tumours, or tumours with nodal involvement (van Hagen et al, 2012). However, in about $20 \%$ of the cases, the tumour is extremely resistant to the preoperative treatment, defined as $>50 \%$ vital residual tumour cells in the surgical specimen, and those patients 
did not benefit from chemoradiotherapy but were exposed to its negative consequences such as toxicity and delayed surgical therapy. These clinical evidences provide a strong argument for the urgent development of molecular biomarkers able to predict primary resistance to preoperative chemoradiotherapy in oesophageal and oesophagogastric junction adenocarcinoma, in order to avoid this preoperative treatment in those patients unlikely to benefit.

Baculoviral inhibitor of apoptosis (IAP) repeat containing (BIRC) 3 gene encodes for the cellular IAP 2 protein (Srinivasula and Ashwell, 2008), a member of the IAP family that inhibits apoptosis by directly inhibiting caspases cascade (Wang et al, 1998; Park et al, 2004). A sequence analysis of the BIRC3 promoter revealed two critical nuclear factor $\kappa \mathrm{B}(\mathrm{NF}-\kappa \mathrm{B})$ and two potential activator protein-1 (AP-1)-binding sites (Hong et al, 2000). Transforming growth factor (TGF)- $\beta$ activated kinase 1 (TAK1, also called MAP3K7) is a serine/threonine kinase with a critical role in the inflammatory responses and cell survival control by integrating signals from various cytokines-including interleukin-1, TGF- $\beta$, and TNF $\alpha$ - and controlling, in turn, the activation of different transcription factors, including AP- 1 and NF- $\kappa$ B (Sakurai, 2012). We recently demonstrated that suppressing the expression of BIRC3 through the genetic silencing or the pharmacological inhibition of TAK1 markedly reverted the intrinsic chemoresistance of pancreatic cancer (Melisi et al, 2011).

In this present study, we hypothesised that the TAK1-regulated expression of BIRC3 might be responsible for the resistance of distal oesophageal and oesophagogastric junction carcinoma to the proapoptotic effect of chemoradiotherapeutic treatments. Thus, the expression of BIRC3 gene might serve as a putative biomarker for the selection of patients with oesophageal and oesophagogastric junction adenocarcinoma eligible for preoperative chemoradiotherapy.

\section{MATERIALS AND METHODS}

Cell lines and reagents. The human distal oesophageal adenocarcinoma cell lines, FLO-1 and KYAE-1, were purchased from European Collection of Cell Cultures (Salisbury, UK). The human pancreatic cancer cell line AsPc-1 was purchased from the American Type Culture Collection (Manassas, VA, USA). AsPC- $1^{\text {shTAK1 }}$ cells stably expressing small hairpin RNA sequences to knockdown the expression of TAK1 has been previously described in Melisi et al (2011). Distal oesophageal adenocarcinoma and pancreatic cancer cell lines were maintained in RPMI 1640 and Dulbecco's modified Eagle's media (LONZA, Basel, Switzerland), respectively, supplemented with $10 \%$ heat-inactivated FBS, $20 \mathrm{mmoll}^{-1} \operatorname{HEPES}\left(\mathrm{pH}\right.$ 7.4), penicillin (100 UI ml ${ }^{-1}$ ), streptomycin $\left(100 \mathrm{mg} \mathrm{ml}^{-1}\right)$, and $4 \mathrm{mmoll}^{-1}$ glutamine (ICN Biomedicals Ltd., Irvine, CA, USA) in a humidified atmosphere of $95 \%$ air and $5 \% \mathrm{CO}_{2}$ at $37^{\circ} \mathrm{C}$. The TAK1 kinase activity was targeted using (5Z)-7-oxozeaenol TAK1 kinase selective inhibitor (TOCRIS bioscience, Bristol, UK). For in vitro assays, (5Z)-7oxozeaenol was dissolved in $100 \%$ dimethyl sulfoxide (DMSO) at a stock concentration of $10 \mathrm{mM}$. Cell irradiation was performed using a GammaCell 40 irradiator (Best Theratronics Ltd., Ottawa, Canada) as previously described in Melisi et al, 2009.

Protein extraction and western blotting. Western blot analyses were performed as previously described in Raben et al (2004). In brief, cell lines were washed twice with cold phosphate-buffered saline and lysed at $4{ }^{\circ} \mathrm{C}$ into radioimmunoprecipitation assay buffer (50 mM Tris- $\mathrm{HCl}$ (pH 8), $150 \mathrm{mM} \mathrm{NaCl}, 1 \%$ Nonidet P-40, $0.5 \%$ sodium deoxycholate, and $0.1 \%$ sodium dodecyl sulfate) plus protease inhibitor mix $(50 \mathrm{nM}$ sodium pyrophosphate, $0,5 \mathrm{mM}$ sodium orthovanadate, $50 \mathrm{mM} \mathrm{NaF}, 5 \mu \mathrm{g} \mathrm{ml}^{-1}$ aprotinin, $0.5 \mathrm{mM}$
PMSF (fenilmetilsulfonil hydrofluoride), $1 \mathrm{mM} \mathrm{DTT,} 2 \mu \mathrm{g} \mathrm{ml}^{-1}$ leupeptin, $5 \mu \mathrm{g} \mathrm{ml}^{-1}$ benzamidine, $1 \mu \mathrm{g} \mathrm{ml}^{-1}$ pepstatine). Lysates were clarified by centrifugation and protein concentrations were determined by BCA assay (Thermo Scientific, Waltham, MA, USA). Each lysate was separated by sodium dodecyl sulfate-polyacrylamide gel electrophoresis and probed $(1: 1000)$ with rabbit monoclonal antibodies against BIRC3, cleaved poly (ADP-ribose) polymerase 1 (PARP1), and cleaved caspase 3 (Cell Signalling Technology, Danvers, MA, USA), or goat polyclonal antibodies against $\gamma$-tubulin (Santa Cruz Biotechnology, CA, USA). Immunoreactive proteins were detected using an enhanced chemiluminescence reagent (Millipore, Billerica, MA, USA) according to the manufacturer's instructions. Images were captured either on film or by LAS4000 Digital Image Scanning System (GE Healthcare, Little Chalfont, UK).

Patients and treatment. Patients considered for analyses were affected by cT2-4, Nx, M0 adenocarcinoma, or squamous cell carcinoma of the distal oesophagus or the oesophagogastric junction (siewert type I and II), and enrolled in a recent study of preoperative therapy with weekly docetaxel and cisplatin, 5-fluorouracil continuous infusion, and concurrent radiotherapy as reported in Pasini et al (2013) and Zanoni et al (2013). Work-up procedures included endoscopic ultrasound, computer tomography (CT) scan chest, abdomen and pelvis, and FDG PET CT scan. Treatment plan was as follows: docetaxel $35 \mathrm{mg} \mathrm{m}^{-2}$ and cisplatin $25 \mathrm{mg} \mathrm{m}^{-2}$ on days $1,8,15,29,36,43,50$, and 57 plus 5-fluorouracil $180 \mathrm{mg} \mathrm{m}^{-2}$ c.i. on days $1-21$ and $150 \mathrm{mg} \mathrm{m}^{-2}$ c.i. on days 29-63. Concurrent RT at $50 \mathrm{~Gy}$ in 25 fractions was started at day 29. Radiological response, according to RECIST criteria v1.1, was assessed by CT, and FDG PET CT scans before therapy and 4 weeks after the end of treatment. Surgery was carried out 6-8 weeks after completion of therapy. The protocol was approved by the ethical committee of the University Hospital of Verona, Italy, and informed consent was obtained from all patients.

Tumour response was evaluated either by Mandard's tumour regression grade (TRG; Mandard et al, 1994; TRG1-3 = responders vs TRG4-5 = non responders) or by size-based pathological response (SPR) classifications (Verlato et al, 2010; SPR1-2= responders $v s \mathrm{SPR} 3-4=$ non responders).

A fresh specimen from the tumour was collected endoscopically at diagnosis and placed immediately into RNALater (Life Technologies, Carlsbad, CA, USA), washed in ice-cold RNAasefree water for $5 \mathrm{~min}$ and 'snap' frozen in liquid nitrogen and stored at $-80^{\circ} \mathrm{C}$ for $24 \mathrm{~h}$.

RNA was isolated by Trizol reagent as indicated by the manufacturer's instructions (Invitrogen, Carlsbad, CA, USA). The reverse transcription-PCR assay was performed as previously described in Rosa et al (2011) accordingly with the high capacity cDNA reverse transcription kit (Applied Biosystems, Foster City, CA, USA). The mRNA expression of BIRC3 was quantified using a SYBR green-based real-time PCR analysis and the ABI Prism 7900 HT Sequence Detection System (Applied Biosystems). Gene expression was tested in each sample in four replicates. To quantify the relative changes in gene expression, the $2^{-\Delta \Delta C T}$ method was used and reactions were normalised to endogenous control gene $\beta$-actin expression levels. The BIRC3 median expression in normal oesophagogastric mucosa samples from six different healthy subjects was used as calibrator (Livak and Schmittgen, 2001).

Statistical analysis. All results were expressed as the mean and 95\% confidence interval for at least three independent experiments performed in triplicate. Drug interactions were studied for synergistic effect according to Chou and Talalay method by CalcuSyn software (Chou and Talalay, 1984). Receiver operator 
characteristic (ROC) analyses were performed to determine the performance of BIRC3 expression levels in distinguishing patients with sensitive or resistant carcinoma. The optimal cutoff thresholds for BIRC3 gene expression were obtained based on the maximisation of the Youden's statistics $J=$ sensitivity + specificity +1 (Youden, 1950) using an R-based software as described in Budczies et al (2012). Statistical analyses were performed using SPSS Statistics 22 (IBM Corporation, Somers, NY, USA), GraphPad Prism software program (version 6.0; GraphPad Software, San Diego, CA), and the statistical language R.

\section{RESULTS}

BIRC3 expression is elevated in distal oesophageal adenocarcinoma cell lines, and regulated by TAK1 kinase activity. In order to demonstrate our hypothesis, we used the two certified distal oesophageal adenocarcinoma FLO-1 and KYAE-1 cell lines (Boonstra et al, 2010), and we initially determined the basal expression of BIRC3 compared with the TAK1-positive pancreatic cancer cell line AsPC-1, as positive control, or the same cells stably silenced for TAK1 expression (Figure 1A). To verify that the inhibition of TAK1 could modulate the expression of BIRC3 in oesophageal adenocarcinoma, we used the selective TAK1 inhibitor (5Z)-7-oxozeaenol, and we demonstrated that the inhibition of TAK1 markedly suppressed the expression of BIRC3 within $24 \mathrm{~h}$ of treatment in both FLO-1 and KYAE-1 distal oesophageal adenocarcinoma cell lines (Figure $1 \mathrm{~B}$ and $\mathrm{C}$ ).

Dowregulating BIRC3 expression through the inhibition of TAK1 sensitises oesophageal adenocarcinoma cell lines to the proapoptotic activity of classic chemotherapeutic agents. To test our hypothesis that TAK1-regulated expression of BIRC3 would be
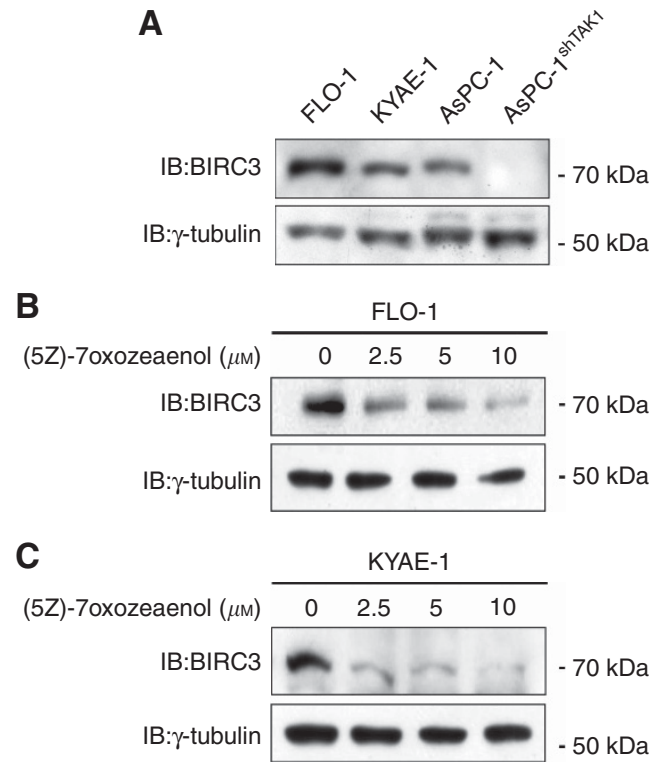

Figure 1. Transforming growth factor- $\beta$ (TGF- $\beta$ )-activated kinase 1 (TAK1) inhibitor (5Z)-7-oxozeaenol downregulates Baculoviral inhibitor of apoptosis (IAP) repeat containing (BIRC)3 in distal oesophageal adenocarcinoma cells. (A) Western blot analysis of BIRC3 basal expression in FLO-1 and KYAE-1 distal oesophageal adenocarcinoma cells compared with AsPC-1 and AsPC-1 1 shTAK1 pancreatic cancer cells as positive and negative control, respectively. (B and C) Western blot analysis of BIRC3 levels after $24 \mathrm{~h}$ of treatment with increasing doses of (5Z)-7-oxozeaenol. $\gamma$-Tubulin was detected as a loading control. responsible for the chemoresistance of distal oesophageal adenocarcinoma, we initially determined the sensitivity of FLO-1 and KYAE-1 cell lines to three chemotherapeutic agents that are currently used for the preoperative treatment of this disease: the nucleoside analogue 5-fluorouracile, the DNA-intercalating agent cisplatin, and the microtubule stabiliser paclitaxel (Supplementary Figure S1). Whereas FLO-1 and KYAE-1 cell lines were protected towards the proapoptotic activity of these chemotherapeutic agents as single-agent treatment or in their triple combination, the inhibition of the expression of BIRC3 by introducing the TAK1 inhibitor (5Z)-7-oxozeaenol was able to modulate their chemoresistance, as demonstrated by significantly higher levels of PARP1 and caspase 3 cleavage (Figure $2 \mathrm{~A}$ and $\mathrm{B}$ ), as well as Annexin- $\mathrm{V}$ positive cells (Figure 2C).

Thus, we combined equitoxic doses of the three chemotherapeutic agents and we measured a statistically significant potentiation of their in vitro antitumour activity when co-administered with increasing doses of TAK1 inhibitor (5Z)-7-oxozeaenol (Figure 2D and E). Moreover, drug interactions were studied for synergistic effect according to Chou and Talalay method (Chou and Talalay, 1984). FLO-1 and KYAE-1 cell lines were treated with increasing doses of 5-fuorouracil, cisplatin, or paclitaxel administered as single agent or in their double or triple combination with or without pretreatment with (5Z)-7oxozeaenol. The pharmacological inhibition of TAK1 induced a strong synergistic antiproliferative effect in all tested combinations and this effect is even more evident when we treated cells with the triple polychemotherapy approach as demonstrated by combination indexes lower than one (Figure $2 \mathrm{~F}$ and G; Supplementary Table S1).

These data suggest that the TAK1-regulated expression of BIRC3 is an important mediator of resistance to chemotherapeutic agents in oesophageal adenocarcinoma in vitro models.

Downregulating BIRC3 expression through the inhibition of TAK1 sensitises oesophageal adenocarcinoma cell lines to radiotherapy. To test our hypothesis that TAK1-regulated expression of BIRC3 would be responsible for the resistance of distal oesophageal adenocarcinoma to radiotherapy, we treated FLO-1 and KYAE-1 cell lines with low doses of (5Z)-7oxozeaenol or DMSO as control plus increasing doses of radiotherapy. The pharmacological inhibition of TAK1 induced a significant downregulation of BIRC3, relieving a crucial cell escape mechanism and thus improving activation of the apoptotic cascade as demonstrated by an increased level of caspase 3 and PARP1 cleavage products and AnnexinV staining in both FLO-1 and KYAE-1 cell lines (Figure 3A, B and C).

These data suggest that the TAK1-regulated expression of BIRC3 could be an important mediator of resistance to radiotherapy in oesophageal adenocarcinoma in vitro models.

Correlation between BIRC3 expression and the response to preoperative chemoradiotherapy treatment in oesophageal and oesophagogastric junction adenocarcinoma and squamous cell carcinoma patients. In order to translate our findings into the clinic setting and evaluate the potential of BIRC3 expression to predict chemoradioresistance in oesophageal and oesophagogastric junction cancers, we measured the expression levels of BIRC3 mRNA in pretreatment biopsies from 32 patients with adenocarcinoma and 33 patients with squamous cell carcinoma treated with a preoperative schedule including weekly docetaxel and cisplatin, continuous infusion of 5-fluorouracil and concomitant radiotherapy as described in Pasini et al (2013). Patient characteristics are shown in Table 1 . The majority of patients were men (86\%). Adenocarcinoma and squamous cell carcinoma histological subtypes were equally represented. Most of the patients had T3 or higher tumours, and half of them had node positive disease. 
A

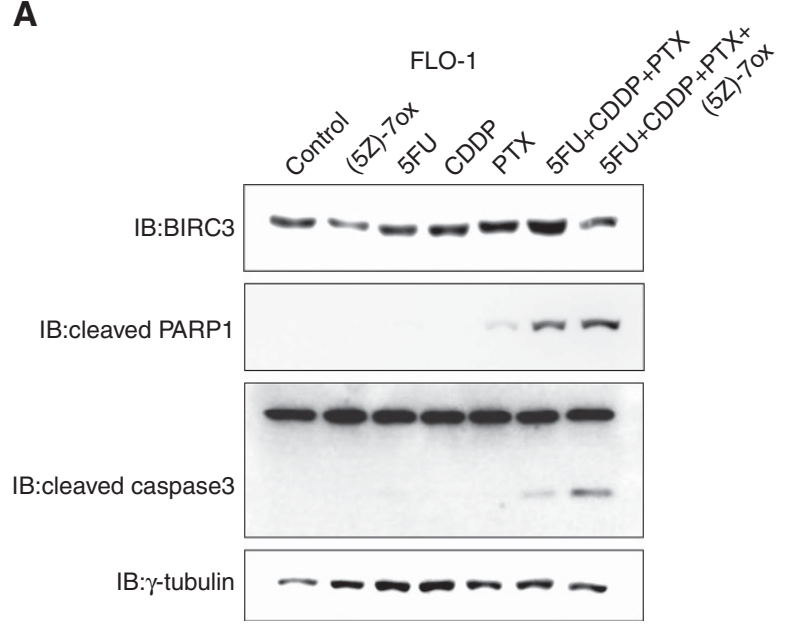

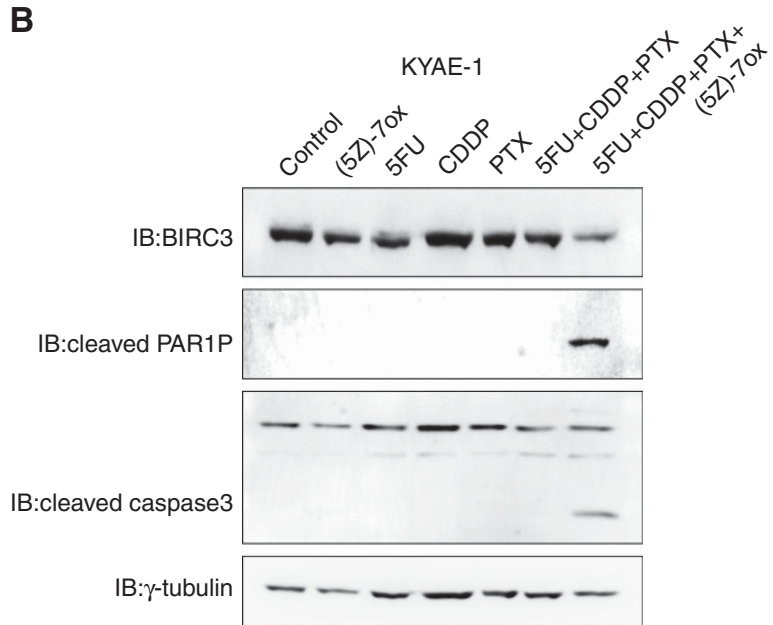

C

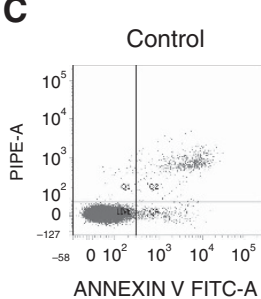

(5Z)-7oxozeaenol
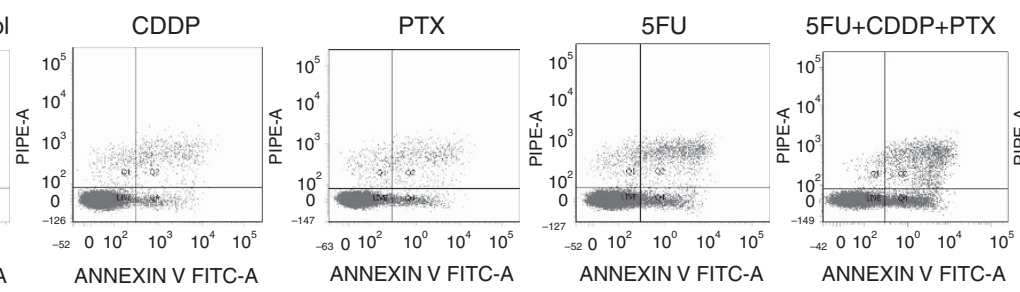

$5 \mathrm{FU}+\mathrm{CDDP}+\mathrm{PTX}+$

(5Z)-7oxozeaenol

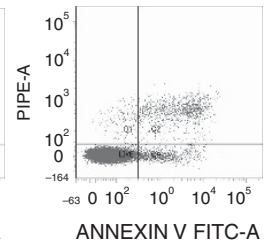

ANNEXIN V FITC-A

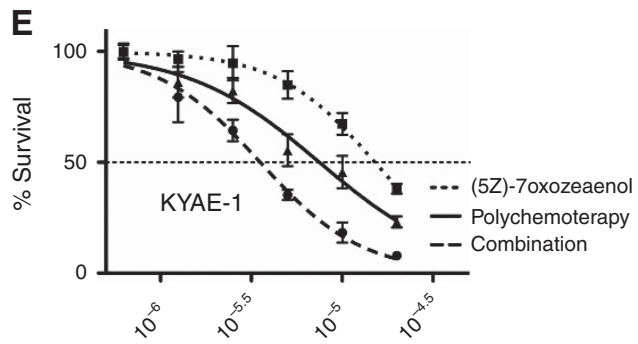

(5Z)-7oxozeaenol (M)

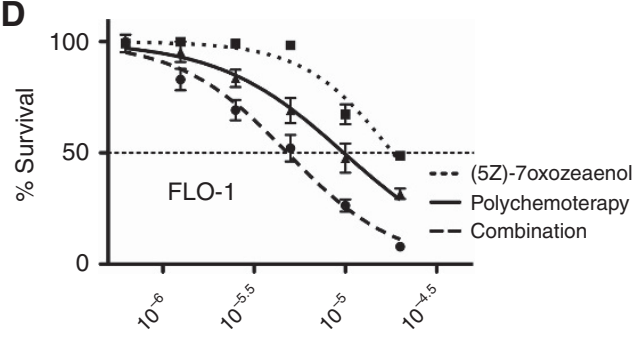

(5Z)-7oxozeaenol (M)

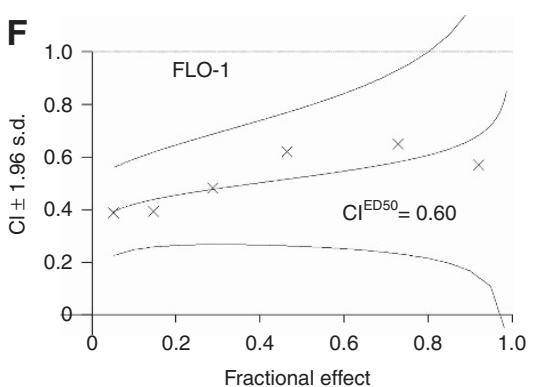

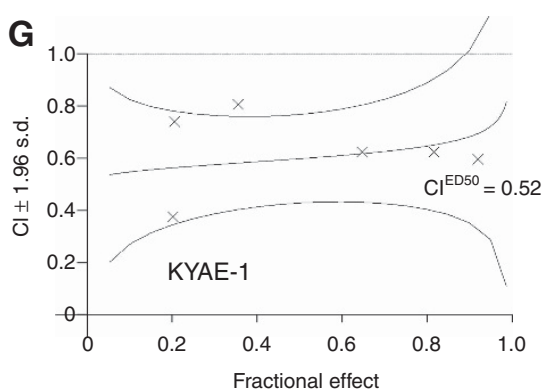

Figure 2. Transforming growth factor- $\beta$ (TGF- $\beta$ )-activated kinase 1 (TAK1) inhibitor-dependent downregulation of Baculoviral inhibitor of apoptosis (IAP) repeat containing (BIRC)3 and chemosensitisation of human oesophageal adenocarcinoma cells in vitro. (A and B) Western blot analysis of BIRC3, cleaved poly (ADP-ribose) polymerase 1 (PARP1) and cleaved caspase 3 levels after $48 \mathrm{~h}$ of treatment with the indicated drugs at their $I_{2}$ doses. $\gamma$-Tubulin was detected as a loading control. (C) The extent of apoptosis was determined after $48 \mathrm{~h}$ of treatment by Annexin $\mathrm{V}$ and propidium iodide staining and flow cytometric analysis. Data shown are representative of three independent experiments. (D and E) FLO-1 and KYAE-1 cells were pretreated with (5Z)-7-oxozeaenol or dimethyl sulfoxide (DMSO) as control for $24 \mathrm{~h}$, and subsequently treated with equitoxic increasing doses of 5-fluorouracil, cisplatin, and paclitaxel (polychemotherapy) for $72 \mathrm{~h}$. Sulforhodamine B (SRB) assay was used to obtain relative estimates of viable cell number. Three independent experiments with four replicates each were performed. Means and $95 \%$ confidence intervals are shown. Curves were fitted by nonlinear regression analysis. (F and $\mathbf{G})$ Combination index plot of (5Z)-7-oxozeaenol in combination with polychemotherapy, representing a quantitative measure of the degree of drug interaction for a given end point of the effect measurement. The combination index $(\mathrm{Cl})$ values of $<1,1$, and $>1$ indicate synergy, additivity, and antagonism, respectively. Data shown are representative of at least three different experiments.

Initially, we demonstrated a significantly lower expression of BIRC3 in the more sensitive population of patients affect by squamous cell carcinoma than in those affected by adenocarcinoma (Figure 4A). Next, we performed ROC analyses to validate the potential usefulness of BIRC3 tumour expression as a biomarker to predict response to preoperative chemoradiotherapy. 
A

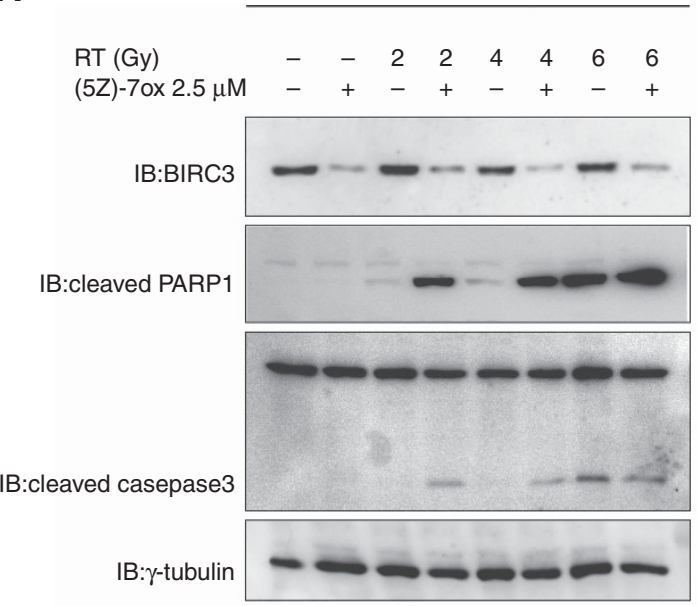

C

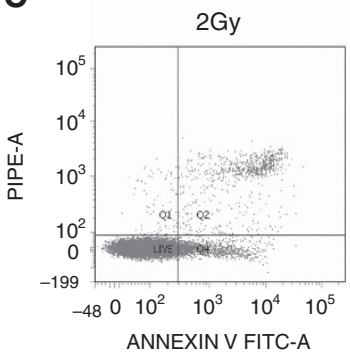

$2 \mathrm{~Gy}+$

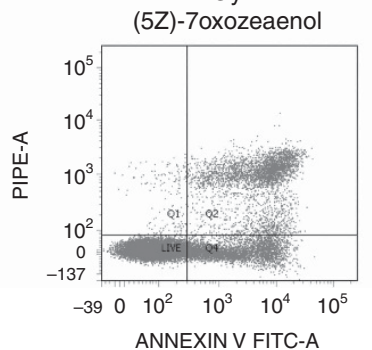

B

FLO-1

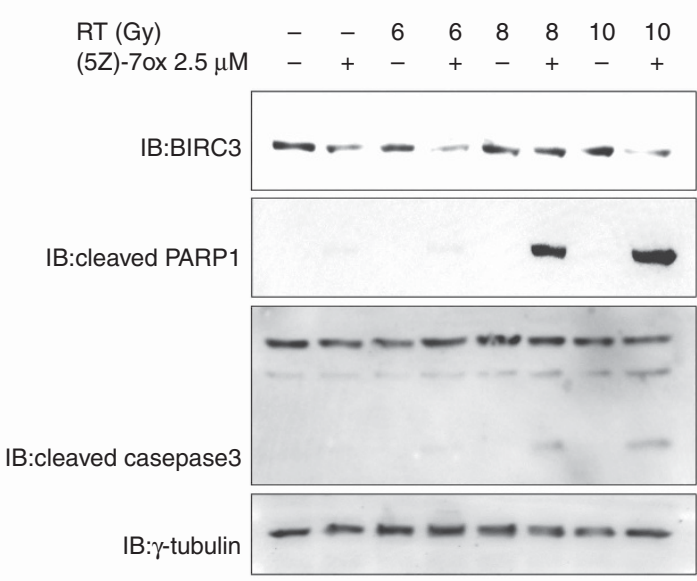

$4 \mathrm{~Gy}+$

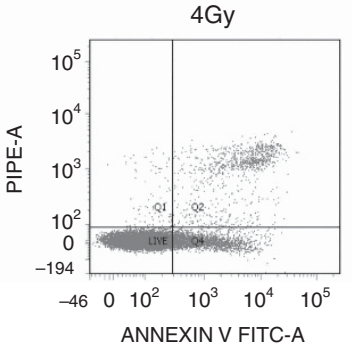

Figure 3. (5Z)-7-oxozeaenol sensitises tumour cells to radiation. (A and B) FLO-1 and KYAE-1 cells were treated with (5Z)-7-oxozeaenol or dimethyl sulfoxide (DMSO) as control, and then irradiated as indicated. Western blot analysis of Baculoviral inhibitor of apoptosis (IAP) repeat containing (BIRC)3, cleaved poly (ADP-ribose) polymerase 1 (PARP1) and cleaved caspase 3 levels was performed after $48 \mathrm{~h}$ form the treatment. $\gamma$-Tubulin was detected as a loading control. (C) The extent of apoptosis was determined after $48 \mathrm{~h}$ of treatment by Annexin $\mathrm{V}$ and propidium iodide staining and flow cytometric analysis. Data shown are representative of three independent experiments.

Whereas tumour expression levels of BIRC3 could not distinguish between sensitive or resistant oesophageal squamous cell carcinoma, it significantly discriminated patients with sensitive or resistant adenocarcinoma, with AUC values of 0.7773 or 0.8074 using the SPR or TRG classifications, respectively (Figure 4B). A $2^{-\triangle \Delta \mathrm{CT}}$ of 2.373 or 4.395 were identified as the optimal cutoff thresholds considering SPR or TRG classifications, respectively. These cutoff thresholds had a sensitivity of $62.5 \%(95 \% \mathrm{CI}=81.5-$ $38.6 \%)$ and specificity of $93.8 \%(95 \% \quad \mathrm{CI}=99.7-71.7 \%)$ by considering the SPR classification, or a sensitivity of $63 \%(95 \%$ $\mathrm{CI}=78.5-44.2 \%)$ and specificity of $100 \%(95 \% \mathrm{CI}=100-56.6 \%)$ by considering the TRG classification for response prediction (Figure 4C). In particular, pathologic complete response was achieved in 6 out of 11 (54.5\%) of patients with BIRC3 expression lower than cutoff, and only in 4 out of $21(19.0 \%)$ of patients with BIRC3 expression higher than cutoff by considering the SPR classification, and in 9 out of 17 (52.9\%) of patients with BIRC3 expression lower than cutoff, and in 6 out of 15 (40\%) of patients with BIRC3 expression higher than cutoff by considering the TRG classification for response prediction (Figure 4D).

\section{DISCUSSION}

In this study, we demonstrated that the suppression of the expression of the antiapoptotic gene BIRC3 regulated by TAK1 significantly increases the sensitivity of oesophageal adenocarcinoma cells to the chemotherapy and radiotherapy-induced cell death. More importantly, we demonstrated that BIRC3 gene expression is a potential biomarker for identifying patients with adenocarcinoma of the distal oesophagus and of the oesophagogastric junction that could most likely benefit from preoperative chemoradiotherapy.

The incidence of the oesophageal adenocarcinoma is rapidly and constantly rising, and the clinical outcomes with current therapeutic approaches remain extremely poor. Thus, there is an increasingly urgent clinical need for developing novel therapeutic strategies able to overcome tumour resistance, as well as for optimising the currently available therapeutic modalities through a more accurate selection of patients in order to avoid the use of ineffective and potentially toxic treatments.

The main obstacle to the clinical efficacy of chemo- and radiotherapy is the pre-existence of or the development of cellular drug resistance (Melisi et al, 2013), a feature that appears to be closely connected to alterations in key pathways involved in cell cycle checkpoint control and, most importantly, apoptosis (DeVita and $\mathrm{Chu}, 2008$ ). A number of recent studies have demonstrated the potential of different molecular markers to predict histopathological response in patients with oesophageal adenocarcinoma receiving preoperative treatments, mostly including growth factor receptors, tumour suppressor genes, and genes associated with chemotherapeutic agents metabolism (reviewed in Bain and Petty (2010)). The TAK1 is a unique member of the MAP3K family (Mihaly et al, 2014), representing the active component of a larger protein signalling complex including TAK1-binding proteins and TRAF6, that integrates several signal transduction cascades, and sustains, in turn, the activation of the most potent antiapoptotic transcriptional factor NF- $\kappa$ B (Melisi and Chiao, 2007; Carbone and Melisi, 2012). We recently demonstrated TAK1 as one of the major 
Table 1. Patients characteristics

\begin{tabular}{|c|c|c|}
\hline Baseline characteristic & $N$ & $\%$ \\
\hline \multicolumn{3}{|l|}{ Age (years) } \\
\hline $\begin{array}{l}\text { Median } \\
\text { Range }\end{array}$ & $\begin{array}{c}62 \\
45-77\end{array}$ & \\
\hline \multicolumn{3}{|l|}{ Gender } \\
\hline $\begin{array}{l}\text { Female, } n(\%) \\
\text { Male, } n(\%)\end{array}$ & $\begin{array}{r}9 \\
56 \\
\end{array}$ & $\begin{array}{l}13.85 \\
86.15 \\
\end{array}$ \\
\hline \multicolumn{3}{|l|}{ Histology } \\
\hline $\begin{array}{l}\text { Adenocarcinoma, n (\%) } \\
\text { Scc, n (\%) }\end{array}$ & $\begin{array}{l}32 \\
33\end{array}$ & $\begin{array}{l}49.23 \\
50.77\end{array}$ \\
\hline \multicolumn{3}{|l|}{ cTumour stage } \\
\hline $\begin{array}{l}\text { T2, n (\%) } \\
\text { T3, n (\%) } \\
\text { T4, n (\%) }\end{array}$ & $\begin{array}{r}2 \\
54 \\
9\end{array}$ & $\begin{array}{r}3.07 \\
83.07 \\
13.84 \\
\end{array}$ \\
\hline \multicolumn{3}{|l|}{ cNodal stage } \\
\hline $\begin{array}{l}\text { No, n (\%) } \\
N+, n(\%)\end{array}$ & $\begin{array}{l}24 \\
41\end{array}$ & $\begin{array}{l}36.92 \\
63.08 \\
\end{array}$ \\
\hline \multicolumn{3}{|l|}{ cMetastasis stage } \\
\hline $\begin{array}{l}M 0, n(\%) \\
M+, n(\%)\end{array}$ & $\begin{array}{r}62 \\
3\end{array}$ & $\begin{array}{r}95.38 \\
4.61 \\
\end{array}$ \\
\hline \multicolumn{3}{|l|}{ cTNM } \\
\hline $\begin{array}{l}\text { cT2N0, n (\%) } \\
\text { cT3N0, n (\%) } \\
\text { cT3N + n (\%) } \\
\text { cT3N + M +, n (\%) } \\
\text { cT4N0, n (\%) } \\
\text { cT4N + } n(\%)\end{array}$ & $\begin{array}{r}2 \\
19 \\
32 \\
3 \\
3 \\
6\end{array}$ & $\begin{array}{r}3.08 \\
29.23 \\
49.23 \\
4.62 \\
4.62 \\
9.23 \\
\end{array}$ \\
\hline \multicolumn{3}{|l|}{ SPR } \\
\hline $\begin{array}{l}1, n(\%) \\
2, n(\%) \\
3, n(\%) \\
4, n(\%)\end{array}$ & $\begin{array}{r}28 \\
9 \\
7 \\
21\end{array}$ & $\begin{array}{l}43.08 \\
13.85 \\
10.77 \\
32.31\end{array}$ \\
\hline \multicolumn{3}{|l|}{ TRG } \\
\hline $\begin{array}{l}1, n(\%) \\
2, n(\%) \\
3, n(\%) \\
4, n(\%) \\
5, n(\%)\end{array}$ & $\begin{array}{r}34 \\
11 \\
6 \\
11 \\
3\end{array}$ & $\begin{array}{r}52.31 \\
16.92 \\
9.23 \\
16.92 \\
4.62\end{array}$ \\
\hline
\end{tabular}

determinant of the unique resistance of pancreatic cancer to the proapoptotic activity of classic chemotherapeutic agents through the transcription of the antiapoptotic gene BIRC3 (Melisi et al, 2011). In this present study, we corroborate these evidences by demonstrating that this role in treatment resistance for the axis TAK1-BIRC3 could also be translated in models of oesophageal adenocarcinoma. Most importantly, our study is the first to demonstrate a significant role for the antiapoptotic gene BIRC3 in predicting histopathological response to preoperative treatments in patients with solid tumours, and, in particular, with oesophageal adenocarcinoma.

The relevance of chemoresistance in oesophageal and oesophagogastric junction adenocarcinoma has been established in a recent analysis involving 400 patients with resectable disease who received preoperative chemotherapy. This study demonstrated that local downstaging after preoperative chemotherapy was the strongest independent predictor of survival in this clinical setting. Tumour stage after preoperative chemotherapy was more relevant for prognosis than initial stage at diagnosis (Davies et al, 2014). In our present study, we analysed the association of BIRC3 expression with patients' survival by considering the established cutoff value of 2.373. Although not statistically significant $(P=0.262)$, we measured a median overall survival (mOS) of 30 vs 64 months for patients with tumour expressing higher or lower levels of BIRC3, respectively (data not shown). Collectively, these evidences corroborate the importance of an early identification of patients who could not benefit from preoperative treatment, and, in turn, the relevance of BIRC3 expression in predicting local downstaging from preoperative treatment in oesophageal and oesophagogastric junction adenocarcinoma.

The most recent and solid evidences in supporting for a preoperative combined chemoradiotherapy followed by surgery approach have been provided by the results of the Chemotherapy for Oesophageal Cancer Followed by Surgery Study (CROSS) (van Hagen et al, 2012). An important question raised by the CROSS trial is the different relevance for preoperative chemoradiotherapy between the two different histological subtypes. For the intention-to-treat population in this study, preoperative chemoradiotherapy provided a $23 \%$ increase in radical resection rate $(92 \%$ vs $69 \%, P<0.001)$, and doubled the mOS duration (49.4 vs 24.0 months, $P<0.003$ ) when compared with surgery. However, in the post hoc analysis, according to histological subtypes, the mOS advantage provided by preoperative chemoradiotherapy was highly significant only for the squamous cell subtype (HR of 0.422 (95\% CI 0.226-0.788)), while among adenocarcinomas the survival gain obtained by the addition of preoperative treatments did not achieve statistical significance (HR of 0.741 (95\% CI $0.536-1.024)$ ), suggesting that the difference between the study arms was driven by the squamous cell subtype despite this being the less numerous group. A pathological complete response to preoperative chemoradiotherapy was obtained in $23 \%$ of adenocarcinomas vs $49 \%$ of squamous cell carcinoma $(P=0.008$; van Hagen et al, 2012). These analyses support a divergent intrinsic biological behaviour, and corroborate the evidences for a different mutational prolife between adenocarcinoma and squamous cell histological subtypes (Agrawal et al, 2012; Dulak et al, 2013; Song et al, 2014). In this regard, we demonstrated a significantly lower expression of BIRC3 in squamous cell carcinomas than in adenocarcinomas, supporting the hypothesis that the expression of this antiapoptotic gene could be a mechanism to explain the different resistance to chemoradiotherapy between the two histological subtypes.

However, this study had some limitations, including its retrospective nature, the limited number of patients analysed, and the lack of an external validation analysis on an independent validation cohort.

In conclusion, this study demonstrates that the reduction of the expression on the antiapoptotic gene BIRC3 through the inhibition of the kinase activity of TAK1 is a valid approach for reverting the resistance of oesophageal adenocarcinoma to chemoradiotherapy. The inhibition of TAK1 deserves further development for the treatment of oesophageal adenocarcinoma. Most importantly, this study candidates BIRC3 as one of the potentially useful predictive marker for discriminating patients with oesophageal and oesophagogastric junction adenocarcinoma who will most likely benefit from preoperative chemoradiotherapy. Further investigations with larger prospective studies in this clinical setting are warranted.

\section{ACKNOWLEDGEMENTS}

Part of the work was performed at the Laboratorio Universitario di Ricerca Medica (LURM) Research Center, University of Verona. This work was supported in part by the Associazione Italiana per la Ricerca sul Cancro (AIRC) Start-Up $\mathrm{n}^{\circ} 10129$, and 5 per mille $\mathrm{n}^{\circ} 10016$ to DM, and by the AIRC grants IG 11930, 5 per mille 12182, 12214, and PRIN n 2009 X23L78_005 to GT. 

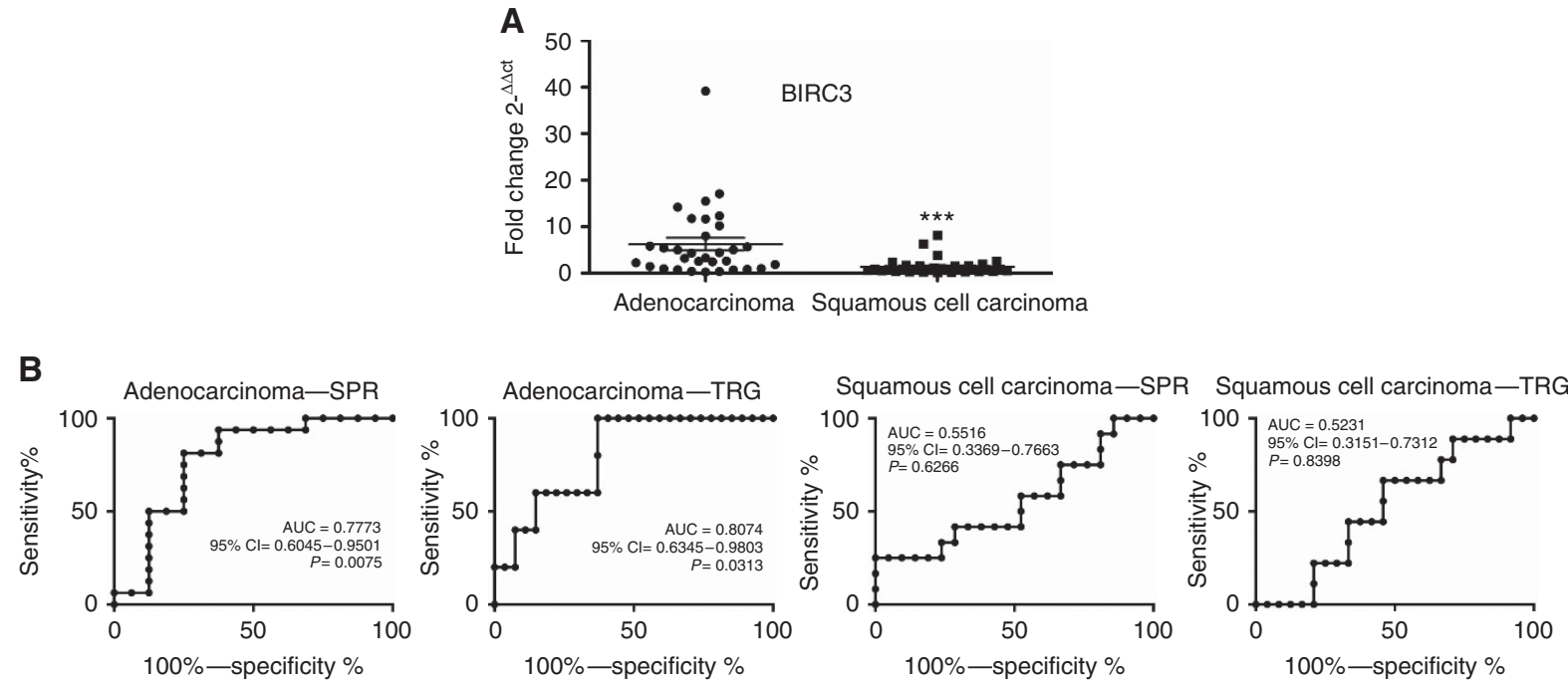

C Significant $(P<0.05)$ tests: 8 out of $13(61.5 \%)$
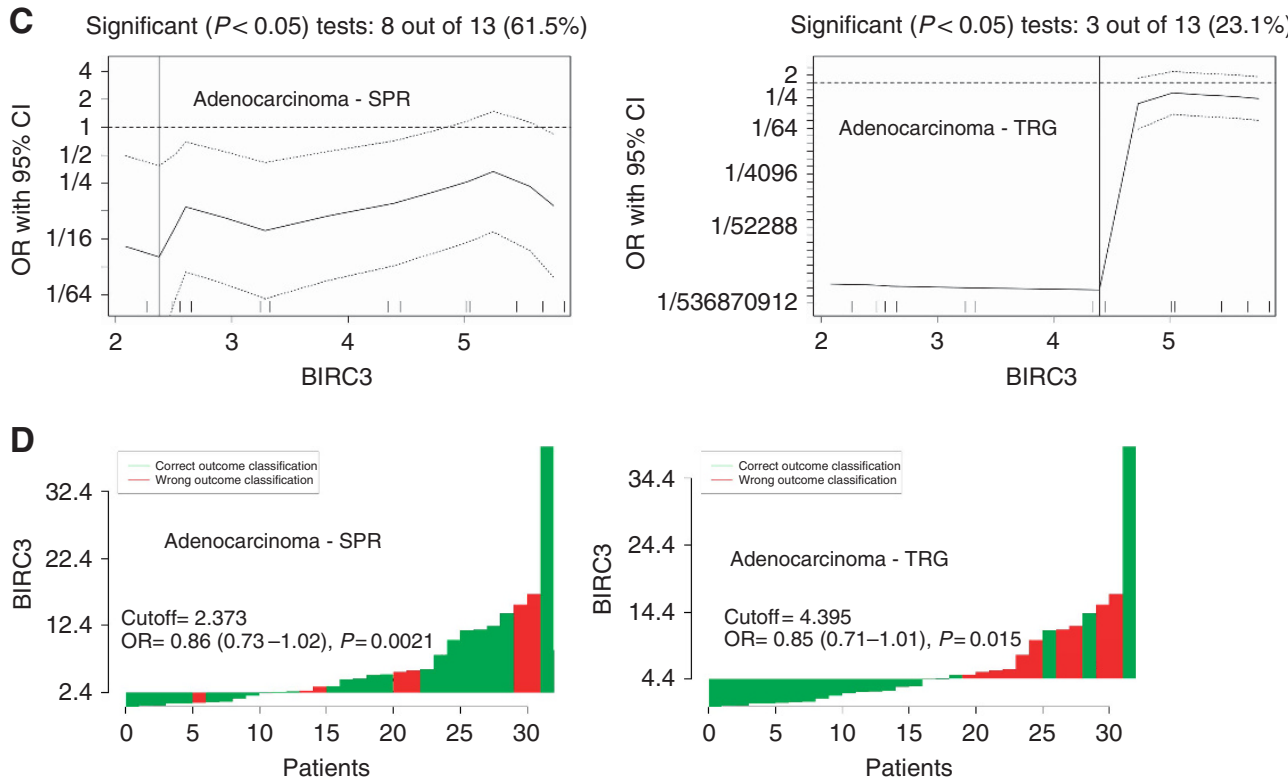

Figure 4. Correlation between Baculoviral inhibitor of apoptosis (IAP) repeat containing (BIRC)3 expression and the response to preoperative chemoradiotherapy treatment in oesophageal and oesophagogastric junction adenocarcinoma and squamous cell carcinoma patients. (A) Distribution of BIRC3 expression in oesophageal adenocarcinomas and squamous cell carcinomas. Horizontal bars denote means. ${ }^{\star * \star} P<0.0001$ by Mann-Whitney test. (B) Receiver operator characteristic (ROC) curves for BIRC3 gene expression in adenocarcinomas and squamous cell carcinomas response evaluated either by Mandard's tumour regression grade (TRG) or by size-based pathological response (SPR) classifications. (C) Determination of cutoff thresholds of BIRC3 gene expression for oesophageal adenocarcinomas response evaluated either by TRG or by SPR classifications. All possible cutoff thresholds were considered and the corresponding odds ratios were calculated and plotted. Each data point in the line gives the corresponding OR and the 95\% confidence interval (dotted lines) on the $y$ axis. Vertical lines indicate the most significant split. (D) Waterfall plot, green and red bars represent cases with correct or wrong classification, respectively.

\section{CONFLICT OF INTEREST}

The authors declare no conflict of interest.

\section{REFERENCES}

Agrawal N, Jiao Y, Bettegowda C, Hutfless SM, Wang Y, David S, Cheng Y, Twaddell WS, Latt NL, Shin EJ, Wang LD, Wang L, Yang W,

Velculescu VE, Vogelstein B, Papadopoulos N, Kinzler KW, Meltzer SJ

(2012) Comparative genomic analysis of oesophageal adenocarcinoma and squamous cell carcinoma. Cancer Discov 2(10): 899-905.
Bain GH, Petty RD (2010) Predicting response to treatment in gastroesophageal junction adenocarcinomas: combining clinical, imaging, and molecular biomarkers. Oncologist 15(3): 270-284.

Boonstra JJ, van Marion R, Beer DG, Lin L, Chaves P, Ribeiro C, Pereira AD, Roque L, Darnton SJ, Altorki NK, Schrump DS, Klimstra DS, Tang LH, Eshleman JR, Alvarez H, Shimada Y, van Dekken H, Tilanus HW, Dinjens WN (2010) Verification and unmasking of widely used human oesophageal adenocarcinoma cell lines. J Natl Cancer Inst 102(4): 271-274.

Budczies J, Klauschen F, Sinn BV, Gyorffy B, Schmitt WD, Darb-Esfahani S, Denkert C (2012) Cutoff Finder: a comprehensive and straightforward Web application enabling rapid biomarker cutoff optimization. PLoS One 7(12): e51862.

Carbone C, Melisi D (2012) NF-kappaB as a target for pancreatic cancer therapy. Expert Opin Ther Targets 16(Suppl 2): S1-S10. 
Chou TC, Talalay P (1984) Quantitative analysis of dose-effect relationships: the combined effects of multiple drugs or enzyme inhibitors. Adv Enzyme Regul 22: 27-55.

Davies AR, Gossage JA, Zylstra J, Mattsson F, Lagergren J, Maisey N, Smyth EC, Cunningham D, Allum WH, Mason RC (2014) Tumor stage after neoadjuvant chemotherapy determines survival after surgery for adenocarcinoma of the oesophagus and esophagogastric junction. J Clin Oncol 32(27): 2983-2990.

DeVita VT, Chu E (2008) Principles of Medical Oncology. In DeVita, Hellman, and Rosenberg's Cancer: Principles \& Practice of Oncology. 8th edn pp 343-346. Wolters Kluwer/Lippincott Williams \& Wilkins: Philadelphia, USA.

Dulak AM, Stojanov P, Peng S, Lawrence MS, Fox C, Stewart C, Bandla S, Imamura Y, Schumacher SE, Shefler E, McKenna A, Carter SL, Cibulskis K, Sivachenko A, Saksena G, Voet D, Ramos AH, Auclair D, Thompson K, Sougnez C, Onofrio RC, Guiducci C, Beroukhim R, Zhou Z, Lin L, Lin J, Reddy R, Chang A, Landrenau R, Pennathur A, Ogino S, Luketich JD, Golub TR, Gabriel SB, Lander ES, Beer DG, Godfrey TE, Getz G, Bass AJ (2013) Exome and whole-genome sequencing of oesophageal adenocarcinoma identifies recurrent driver events and mutational complexity. Nat Genet 45(5): 478-486.

Hong SY, Yoon WH, Park JH, Kang SG, Ahn JH, Lee TH (2000) Involvement of two NF-kappa B binding elements in tumor necrosis factor alpha -, CD40-, and epstein-barr virus latent membrane protein 1-mediated induction of the cellular inhibitor of apoptosis protein 2 gene. J Biol Chem 275(24): 18022-18028.

Livak KJ, Schmittgen TD (2001) Analysis of relative gene expression data using real-time quantitative PCR and the 2(-Delta Delta C(T)) Method. Methods 25(4): 402-408.

Mandard AM, Dalibard F, Mandard JC, Marnay J, Henry-Amar M, Petiot JF, Roussel A, Jacob JH, Segol P, Samama G (1994) Pathologic assessment of tumor regression after preoperative chemoradiotherapy of esophageal carcinoma. Clinicopathologic correlations. Cancer 73(11): 2680-2686.

Melisi D, Chiao PJ (2007) NF-kappa B as a target for cancer therapy. Expert Opin Ther Targets 11(2): 133-144.

Melisi D, Ossovskaya V, Zhu C, Rosa R, Ling J, Dougherty PM, Sherman BM, Abbruzzese JL, Chiao PJ (2009) Oral poly(ADP-ribose) polymerase-1 inhibitor BSI-401 has antitumor activity and synergizes with oxaliplatin against pancreatic cancer, preventing acute neurotoxicity. Clin Cancer Res 15(20): 6367-6377.

Melisi D, Piro G, Tamburrino A, Carbone C, Tortora G (2013) Rationale and clinical use of multitargeting anticancer agents. Curr Opin Pharmacol 13(4): 536-542.

Melisi D, Xia Q, Paradiso G, Ling J, Moccia T, Carbone C, Budillon A, Abbruzzese JL, Chiao PJ (2011) Modulation of pancreatic cancer chemoresistance by inhibition of TAK1. J Natl Cancer Inst 103(15): 1190-1204.

Mihaly SR, Ninomiya-Tsuji J, Morioka S (2014) TAK1 control of cell death. Cell Death Differ 21(11): 1667-1676.

Park SM, Yoon JB, Lee TH (2004) Receptor interacting protein is ubiquitinated by cellular inhibitor of apoptosis proteins (c-IAP1 and c-IAP2) in vitro. FEBS Lett 566(1-3): 151-156.

Pasini F, de Manzoni G, Zanoni A, Grandinetti A, Capirci C, Pavarana M, Tomezzoli A, Rubello D, Cordiano C (2013) Neoadjuvant therapy with weekly docetaxel and cisplatin, 5-fluorouracil continuous infusion, and concurrent radiotherapy in patients with locally advanced esophageal cancer produced a high percentage of long-lasting pathological complete response: a phase 2 study. Cancer 119(5): 939-945.

Raben D, Bianco C, Damiano V, Bianco R, Melisi D, Mignogna C, D’Armiento FP, Cionini L, Bianco AR, Tortora G, Ciardiello F, Bunn P (2004) Antitumor activity of ZD6126, a novel vascular-targeting agent, is enhanced when combined with ZD1839, an epidermal growth factor receptor tyrosine kinase inhibitor, and potentiates the effects of radiation in a human non-small cell lung cancer xenograft model. Mol Cancer Ther 3(8): 977-983.

Rosa R, Melisi D, Damiano V, Bianco R, Garofalo S, Gelardi T, Agrawal S, Di Nicolantonio F, Scarpa A, Bardelli A, Tortora G (2011) Toll-like receptor 9 agonist IMO cooperates with cetuximab in K-ras mutant colorectal and pancreatic cancers. Clin Cancer Res 17(20): 6531-6541.

Sakurai H (2012) Targeting of TAK1 in inflammatory disorders and cancer. Trends Pharmacol Sci 33(10): 522-530.

Siegel R, Ma J, Zou Z, Jemal A (2014) Cancer statistics, 2014. CA Cancer J Clin 64(1): 9-29.

Song Y, Li L, Ou Y, Gao Z, Li E, Li X, Zhang W, Wang J, Xu L, Zhou Y, Ma X, Liu L, Zhao Z, Huang X, Fan J, Dong L, Chen G, Ma L, Yang J, Chen L, He M, Li M, Zhuang X, Huang K, Qiu K, Yin G, Guo G, Feng Q, Chen P, Wu Z, Wu J, Ma L, Zhao J, Luo L, Fu M, Xu B, Chen B, Li Y, Tong T, Wang M, Liu Z, Lin D, Zhang X, Yang H, Wang J, Zhan Q (2014) Identification of genomic alterations in oesophageal squamous cell cancer. Nature 509(7498): 91-95.

Srinivasula SM, Ashwell JD (2008) IAPs: what's in a name? Mol Cell 30(2): 123-135.

van Hagen P, Hulshof MC, van Lanschot JJ, Steyerberg EW, van Berge Henegouwen MI, Wijnhoven BP, Richel DJ, Nieuwenhuijzen GA, Hospers GA, Bonenkamp JJ, Cuesta MA, Blaisse RJ, Busch OR, ten Kate FJ, Creemers GJ, Punt CJ, Plukker JT, Verheul HM, Spillenaar Bilgen EJ, van Dekken H, van der Sangen MJ, Rozema T, Biermann K, Beukema JC, Piet AH, van Rij CM, Reinders JG, Tilanus HW, van der Gaast A. Group C (2012) Preoperative chemoradiotherapy for oesophageal or junctional cancer. N Engl J Med 366(22): 2074-2084.

Verlato G, Zanoni A, Tomezzoli A, Minicozzi A, Giacopuzzi S, Di Cosmo M, Franceschetti I, de Manzoni G (2010) Response to induction therapy in oesophageal and cardia carcinoma using Mandard tumour regression grade or size of residual foci. Br J Surg 97(5): 719-725.

Wang CY, Mayo MW, Korneluk RG, Goeddel DV, Baldwin Jr AS (1998) NF-kappaB antiapoptosis: induction of TRAF1 and TRAF2 and c-IAP1 and c-IAP2 to suppress caspase-8 activation. Science 281(5383): 1680-1683. Youden WJ (1950) Index for rating diagnostic tests. Cancer 3(1): 32-35. Zanoni A, Verlato G, Giacopuzzi S, Weindelmayer J, Casella F, Pasini F, Zhao E, de Manzoni G (2013) Neoadjuvant concurrent chemoradiotherapy for locally advanced esophageal cancer in a single high-volume center. Ann Surg Oncol 20(6): 1993-1999.

This work is published under the standard license to publish agreement. After 12 months the work will become freely available and the license terms will switch to a Creative Commons AttributionNonCommercial-Share Alike 4.0 Unported License.

Supplementary Information accompanies this paper on British Journal of Cancer website (http://www.nature.com/bjc) 